\title{
Drivers' Perceived Workload When Driving Using Adaptive Equipment: A Pilot Study
}

\author{
Dana Benoit, Isabelle Gélinas, Barbara Mazer, Michelle M. Porter \& Josée \\ Duquette
}

To cite this article: Dana Benoit, Isabelle Gélinas, Barbara Mazer, Michelle M. Porter \& Josée Duquette (2009) Drivers' Perceived Workload When Driving Using Adaptive Equipment: A Pilot Study, Physical \& Occupational Therapy In Geriatrics, 27:4, 277-297, DOI: 10.1080/02703180902768650

To link to this article: https://doi.org/10.1080/02703180902768650

曲 Published online: 13 Aug 2009.

Submit your article to this journal $\sqsubset$

Шll Article views: 216

Q View related articles $\square$

4 Citing articles: 4 View citing articles ๘ 


\title{
Drivers' Perceived Workload When Driving Using Adaptive Equipment: A Pilot Study
}

\author{
Dana Benoit, BSc (OT), CDRS, MSc (Rehab Science) \\ Isabelle Gélinas, $\mathrm{BSc}(\mathrm{OT}), \mathrm{PhD}$ \\ Barbara Mazer, BSc (OT), PhD \\ Michelle M. Porter, PhD (Kinesiology) \\ Josée Duquette, BSc (ergothérapie), MSc
}

\begin{abstract}
Objectives: (1) To examine the impact of introducing an adaptive driving device (hand-control) to activate the gas and brake on perceived workload while driving, (2) to determine if age is associated with change in workload when introducing hand-controls, and (3) to investigate the association between perceptual-cognitive status and demographic factors on change in workload when hand-controls are introduced. Methods: Healthy younger $(n=27)$ and older $(n=27)$ drivers were compared on their perceived workload using the NASA Task Load
\end{abstract}

Dana Benoit is a Occupational Therapist, Centre de réadaptation ConstanceLethbridge Faculty Lecturer, McGill University, School of Physical and Occupational Therapy Montreal, Quebec, Canada

Isabelle Gélinas and Barbara Mazer are Associate Professors, McGill University School of Physical and Occupational Therapy, Montreal, Quebec, Canada, Research Associate, Centre de Recherche Interdisciplinaire en Réadaptation du Montréal (CRIR), Jewish Rehabilitation Hospital, Laval, Quebec, Canada

Michelle M. Porter is a Professor, Faculty of Physical Education and Recreation Studies, University of Manitoba, Winnipeg, Canada

Josée Duquette is a Clinical Research Coordinator, CRIR - Centre de réadaptation Constance-Lethbridge, Montréal, Qc, Canada

Address correspondence to: Dana Benoit, BSc (OT), MSc (Rehab Science), CDRS Occupational Therapist, Centre de réadaptation Constance-Lethbridge 7005 Blvd. DeMaisonneuve Ouest, Montreal, Quebec, Canada (E-mail: danabenoit@ssss.gouv.qc.ca dana.benoit@mcgill.ca)

Physical \& Occupational Therapy in Geriatrics, Vol. 27(4), 2009

Available online at http://www.informaworld.com/POTG

(C) 2009 by Informa Healthcare USA, Inc. All rights reserved. doi: 10.1080/02703180902768650 
Index when driving the same route with normal vehicle controls and using hand-controls to activate the gas and brake. Results: Modifying a vehicle's controls significantly increased the demands of the driving task $(p<.0001)$. Healthy older drivers responded similarly to younger drivers, however, seniors with greater attentional declines experienced more difficulties. Women's perceived workload was greater than men's when hand-controls were introduced $(p=.01)$. Conclusions: The results suggest that the introduction of driving adaptations may increase task demands in clients with physical impairments.

KEYWORDS. Adaptive vehicle controls, driver rehabilitation, driver training

\section{INTRODUCTION}

The ability to drive is considered a necessity by many individuals and is strongly associated with well being, mobility, and autonomy (O'Neill, Bruce, Kirby, \& Lawlor, 2000; Persson, 1993). In fact, driving contributes to our independence to such a degree, that those who face driving curtailment are often more concerned about driving restrictions than any other functional limitation (Hopewell, 2002). As such, resumption of driving is a primary objective for clients within the context of rehabilitation.

Occupational therapists working in the field of driver rehabilitation assess the perceptual, cognitive, and physical abilities required for the driving task to identify impairments that may impede a client's ability to safely return to driving. These results are then validated via an onroad driving performance assessment so that the impact of underlying cognitive and/or perceptual deficits on functional performance can be measured. Clients presenting with physical impairments are introduced to various adaptive driving devices in an effort to compensate for limited capacities in strength, endurance, or range of motion in the extremities, torso, or neck. The evaluation process, therefore determines not only whether a client has the perceptual and cognitive abilities to drive safely, it also serves to recommend which vehicle modifications and adaptive devices are most appropriate to render the driving task accessible (Mazer, Gélinas, \& Benoit, 2004). Adaptive equipment includes devices such as left-foot accelerators, hand-controls to activate the gas and brake, reduced-diameter steering wheels, ramps, lifts, and wheelchair loading devices. 
Despite predictions of an increase in the use of adaptive driving aids (Turnbull \& McKenzie, 1997), there remains a lack of research targeting the use of automotive technology for drivers with physical disabilities (Henriksson \& Peters, 2004; Monga, 1997). Consequently, the implementation of adaptive devices and subsequent training for drivers is approached in an intuitive and ad hoc manner (Peters, 2001), rendering this aspect of the intervention process highly variable between rehabilitation centers and clinicians.

Changing demographics in North America project an unprecedented proportion of older persons comprising the driving population in the early 21 st century (NHTSA, 2003). This has led to an abundance of research in the crash-risk analysis of drivers presenting perceptual and/or cognitive decline (Ball, Owsley, Sloane, Roenker, \& Bruni, 1993; Dulisse, 1997; Lyman, Ferguson, Braver, \& Williams, 2002). However, drivers with purely physical functional limitations have not been well studied, as it is usually possible to adapt a vehicle to accommodate older adults with limited mobility (Tuokko \& Hunter, 2002).

The normal aging process results in various functional losses, including decreased speed of information processing (Golde, 2001), reduced attentional skills (Anderson, 1999) and a slowing of decisional and mental processes (Mazer et al., 2004). It has been widely hypothesized that older, very experienced drivers are able to compensate for these changes due to the automaticity of the driving task that has developed over many years (DeWaard, 1996; O'Neill, 2002; Summala, 1988). It is unknown whether altering the operational components of the task (i.e., using adaptive equipment to modify the activation of the gas, brake, or steering of a vehicle) has an effect on the "automaticity" of driving. If the automaticity of the task is altered, are the effects of this different for younger drivers versus their older counterparts?

The emergence of cellular phones and in-vehicle telematics has brought the concept of workload to the foreground in recent automotive literature (Harbluk \& Noy, 2002; Lansdown, Brook-Carter, \& Kersloot, 2004; Peters, 2001; Recarte \& Nunes, 2003). Workload is the term used to indicate how much effort and attention are required to acquire a certain level of performance in a given task (Verway, 1992). Although increasing workload may not necessarily decrease on-road performance, it has been shown that individuals are more likely to be involved in traffic crashes when they are under emotional or internal stress (Legree, Heffner, Psotka, Martin, \& Medsker, 2003). The reporting of perceived workload provides information with respect to a driver's anxiety or comfort level while performing a task, reflecting the degree to which the driver feels challenged. This is an 
important consideration with respect to the driving task. For example, a novice driver who is merging onto a highway for the first time is likely to feel very nervous and uncertain as he or she attempts to blend into the fast-approaching traffic. The maneuver may be performed correctly, but will likely be at the limit of capacity. Measuring this driver's perception of the demands of the maneuver will discriminate him from another, more experienced driver who may have performed the task with comparable skill but under much less strain. The novice driver, if performing at the limit of his capacities, will not have any resources available to respond to an unpredicted event within the traffic environment (e.g., a stalled car in front of him). The measurement of a driver's perception of the task therefore has important connotations regarding the availability of additional resources should a hazardous event occur.

Altering the mechanisms to operate a vehicle by introducing adaptive driving equipment returns an experienced driver back to the novice driver's seat, where a conscience effort is required to remember how to brake or to accelerate. The attentional demands may increase substantially while a driver learns to integrate an unfamiliar device into his driving habits. In addition, due to age-related functional changes at the perceptual and cognitive level, the senior driver may experience greater demands than his younger counterpart.

Rehabilitation professionals must understand the impact of introducing unfamiliar devices on the driver, as well as the age-related differences that may influence the safe integration of this equipment. This study investigated the impact of introducing an adaptive driving device on drivers' perceived workload. The objectives were to examine the change in perceived workload when an adaptive driving device (hand-control) is introduced to the driving task and to determine if age is associated with changes in perceived workload. The association between perceptual-cognitive status (attention, information processing speed and useful field of view) and demographic factors (gender, driving experience, and driving frequency) on change in workload were also investigated.

\section{METHODS}

\section{Participants}

Two groups of subjects participated in this study. Twenty-seven healthy older drivers (60 years + ) and 27 healthy younger drivers (25-45 years) were recruited by word-of-mouth referrals. Although adaptive driving 
devices are implemented when drivers present with physical impairments, nonimpaired drivers were targeted to enable the use of both an adapted vehicle and a nonadapted vehicle. Participants required a valid driver's license and regularly drove cars without adaptive equipment. Older drivers required a minimum of 20 years driving experience, while the younger drivers had to have a minimum of 5 years driving experience. Written informed consent was obtained and participants were advised that they could withdraw from the study at any time. All volunteers were able to communicate in either English or French.

Exclusion Criteria: Drivers between the ages of 46 and 59 were excluded from the study, as this is the gray zone where age-related impairments may or may not be evident, given the variability in the onset of decline due to normal aging (Perez Riley, 1994). Persons within this age range may not yet present with age-related impairments, which would make it difficult to classify them into either the "Younger" or the "Older" group. Volunteers whose health conditions would adversely affect their driving or learning ability (determined by questioning medical status and history) and those presenting underlying perceptual or cognitive deficits (determined by scores below 32 on the Digit Symbol Substitution Test) were also excluded from the study. Finally, participants with past or current vocational driving experience (e.g., taxi drivers, driving instructors, couriers) were excluded as this subset of drivers may respond differently to the introduction of handcontrols than the general population because of their extensive driving exposure.

\section{Procedures}

Following ethical approval, the principle investigator contacted interested volunteers by phone to describe the study and to determine eligibility. Consent forms were sent to potential candidates one week prior to the initial evaluation. Participation in the study involved three phases. In phase one, after consent was obtained, an in-clinic, pre-road assessment was administered by an experienced Occupational Therapist (OT) to assess the cognitive/perceptual status of the participants. This included three evaluations: Trail Making Test-Part A and B, Useful Field of View Test, and Digit Symbol Substitution Test. At this time, information was also collected on sociodemographic characteristics, driving habits, frequency and experience. Phase two immediately followed phase one. Participants were accompanied to an empty parking lot, introduced to the driving instructor, and completed a 30-kilometer (18 mile) driving route in an automatic car 
equipped with a dual brake. During this evaluation, participants' perceived workload during "normal driving" was assessed to provide a baseline measure for comparative purposes. Workload ratings were recorded (using the NASA Task Load Index) at four specific locations during the route following driving segments that differed in their complexity (segment A comprised parking lot maneuvers; segment B included residential driving; segment $C$ progressed to increased speed zones including a highway segment; and segment D introduced busy city driving). The OT sat in the rear seat and recorded traffic conditions, unplanned events and administered the NASA TLX following each driving segment. In phase three, upon return to the empty parking lot, hand-controls were installed to allow activation of the gas and brake using the left upper extremity. As the steering wheel was manipulated unilaterally, a spinner knob was also installed (at the 2 o'clock position) to facilitate turning maneuvers. Participants first sat in the front passenger seat of the vehicle and observed the instructor driving with the adaptive devices for 2-3 min. They then returned to the driving position and practiced turning, accelerating and braking, using the hand-control and spinner knob to accelerate, brake, and steer. After 5 min of practice, the second driving session (time 2) was commenced. The procedures used during this session were identical to those of the first driving session, with the exception that participants drove using the hand-control to operate the gas and brake. Stops were made at the same four locations to complete the workload rating scale.

Since drivers under increased cognitive load tend to slow down and to apply increased brake pressures (Lansdown et al., 2004), vehicle speed variations, incidences of hard accelerations, and incidences of hard braking were recorded using the Davis Carchip ${ }^{\mathrm{TM}}$, a vehicle monitoring system that records speed at $5 \mathrm{~s}$ intervals (Huebner, Porter, \& Marshall, 2006).

\section{Measures}

Perceptual and Cognitive Tests

An OT administered three short perceptual/cognitive assessments: The Trail Making Test (parts A \& B), the Digit Symbol Substitution Test (subtest of the Wechsler adult intelligence scale-R) and the Useful Field of View Test. These measures were chosen because they are commonly used in the clinical assessment of driving ability, are quick to administer, and provide age-related normative data. 
The Trail Making Test (parts A \& B) (Spreen \& Strauss, 1991) is a quick neuropsychological assessment requiring visual search, attention and mental flexibility. Scores are recorded as the amount of time required to complete each of the two tasks (A \& B). Both parts of the test can be administered in less than 5 min. The Trail Making Test (parts A \& B) has good reliability ( $r=0.79$ and 0.89 , respectively) (Dickman, Heaton, Grant, \& Temkin, 1999) and is sensitive to detecting general brain impairment (Drane, Yuspeh, Huthwaite, \& Klingler, 2002).

The Digit Symbol Substitution Test (DSST), a subtest of the Wechsler adult intelligence scale-revised (Wechsler, 1981), is a timed test involving the pairing of numbers with corresponding symbols according to a code provided to the participant. Subjects must identify the symbol that corresponds to each target number by referring to a reference grid of digit/symbol pairings. The score is recorded as the number of correctly completed symbols in $90 \mathrm{~s}$. This test is commonly administered in neuropsychological and epidemiological contexts to assess sustained attention and information processing speeds (Parkin \& Java, 1999; Pavlik et al., 2003). The DSST demonstrates high reliability ( $r=0.89$ ) (Dickman et al., 1999) and is one of the most widely used instruments in cognitive aging studies to describe performance of younger and older adults. A recent meta-analysis of the DSST reported that the speed of carrying out the combination of coding and substitution processes that comprise DSST performance is slower for older adults compared to younger adults and that the age-DSST relationship was independent of years of education (Hoyer, Stawski, Wasylyshyn, \& Verhaeghen, 2004).

The Useful Field of View (UFOV) Visual Attention Analyzer (Ball et al., 1993 ) is a measure of the size of the visual area within which a briefly exposed target can capture one's attention. It is defined as the region of the visual field from which an observer can capture information at any one time. This microprocessor-based instrument comprises three tasks measuring (1) speed of visual processing, (2) ability to divide attention, and (3) selective attention abilities. Results from these subtests combine to provide a level of risk (categorically rated 1-6) associated with driving safety. In a study examining the effects of aging on UFOV $(N=176)$, it was found that performance on the UFOV, including both central target identification and peripheral target localization, deteriorates with age (Sekuler, Bennett, \& Mamelak, 2000). Drivers with a 40\% reduction in their useful field of view are 2.3 times more likely to be involved in atfault crashes than those with no visual attention impairment (Owsley et al., 1998). 
The NASA Task Load Index (TLX) (Hart \& Staveland, 1988) was selected as the primary outcome measure. It is a standardized multidimensional subjective rating scale that uses six dimensions to assess overall mental workload: mental demand, physical demand, temporal demand, performance, effort, and frustration. The NASA TLX is one of the most widely used tools to assess mental workload in human factors research and has been employed in research conducted with older adults (Tomporowski, 2003). It is sensitive to changes in the difficulty of a task and demonstrates a higher correlation with performance variations in a task than do other workload rating scales (Rubio, Diaz, Martin, \& Puente, 2004).

The NASA TLX allows for the addition of separate factors for analysis. Given the task of interest (driving in traffic), it was felt that the participants' feeling of safety was a pertinent issue. Perceived safety therefore replaced the dimension of "effort" on the rating form. A twenty-step bipolar scale was used for each of the six dimensions of the index. Each of the six subscales were rated on a $50 \mathrm{~mm}$ line anchored with the terms "Low" and "High." Participants were instructed to place a mark on the line corresponding to their perception of each dimension; definitions of the subscales were provided while the participant completed the NASA TLX. A score of 0-100 was obtained on each scale (rounded-up to the nearest 5 points). Although the protocol suggests a weighting procedure prior to combining the six subscale ratings into one global score, this was found to be a frustrating task for the first three participants as they had difficulty differentiating the extent to which each dimension contributed to their overall impressions of task-load. The weighting procedure did little to alter the raw workload scores in these participants. Traditional TLX scores (weighted) have been found to correlate almost perfectly ( $r=0.96-$ 0.98 ) with raw scores based on equal weighting (Hill, Iavecchia, Byers, \& Bittner, 1992). Nygren (1991) also suggested that the weighting procedure is "ineffective and should generally be ignored." Ratings for the six subscales of the NASA TLX were therefore combined using equal weighting to produce the overall workload score.

Davis Carchip: Data regarding vehicle control was collected by the Davis Carchip ${ }^{\mathrm{TM}}$ a vehicle monitoring system that records speed at $5 \mathrm{~s}$ intervals, as well as other parameters such as braking and accelerating pressure. Average speeds were calculated for each of the four segments within the route as drivers may have slowed down if workload was perceived to be quite high. Increased frequency of hard braking is another driving behavior observed as a consequence of increased cognitive load while driving (Harbluk \& Noy, 2002). The number of "hard braking" and 
"hard acceleration" events were therefore also calculated for each driving segment.

\section{Data Analysis}

Basic descriptive statistics were used to describe the sociodemographic and clinical characteristics of the two groups of participants as well as the scores obtained on the outcome variables. Several outliers in the NASA TLX scores resulted in substantially large standard deviations, violating the assumption of normalcy. Consequently, the data were analyzed using nonparametric testing procedures to compare ranks, using $p<.05$ as the criterion level of significance.

The Wilcoxon signed rank sum test was used to determine the impact of introducing hand-controls: Grouping younger and older drivers together, each group's mean rating of perceived workload while driving with the adaptive equipment was compared to the reported baseline workload score (i.e., normal driving). This same procedure was applied to analyze average speed variations between the two driving conditions in each segment of the route (continuous data). Dichotomizing the data for age, workload ratings were then analyzed comparing the change in workload between normal driving and driving with adaptive equipment for the older and the younger drivers using the Wilcoxon Mann-Whitney test. This test was also used to compare change in workload for male and female participants.

Associations between perceived workload and sociodemographic characteristics (driving frequency and driving experience) as well as perceptual/cognitive status were analyzed using Spearman correlation tests when data were continuous (scores on DSST, Trail Making A \& B, UFOV subtests and years driving experience). The Kruskal-Wallis test was used for categorical data (driving frequency represented by mileage per week).

\section{RESULTS}

The demographic characteristics of the study participants and their mean scores on the pre-road tests are presented in Table 1. Within the younger drivers, only four participants were aged 25-35 years, while the remainder were between 36 and 45 years. The vast majority of the older group was 60-69 years of age. Five participants were between 70 and 80 years, and one participant was a 91-year-old. As expected, the older group demonstrated longer processing speeds compared to the younger cohort on each 
TABLE 1. Sociodemographic and Clinical Characteristics of the Sample Population

\begin{tabular}{lccc}
\hline & $\begin{array}{c}\text { ALL }(N=54) \\
N(\%)\end{array}$ & $\begin{array}{c}\text { Young }(N=27) \\
N(\%)\end{array}$ & $\begin{array}{c}\text { Old }(N=27) \\
N(\%)\end{array}$ \\
\hline $\begin{array}{l}\text { Gender } \\
\bullet \text { Male }\end{array}$ & & \\
- Female & $25(46.3)$ & $11(40.7)$ & $14(51.8)$ \\
& $29(53.7)$ & $16(59.3)$ & $13(48.2)$ \\
\hline & Mean (SD) & Mean (SD) & Mean (SD) \\
\hline Age & $62.5(13.0)$ & $39.8(5.7)$ & $67.2(6.9)$ \\
$\bullet$ Male & $56.9(15.4)$ & $41.4(3.6)$ & $69.1(7.9)$ \\
- Female & $50.6(14.6)$ & $38.8(6.7)$ & $65.1(5.0)$ \\
Schooling (years) & & $16.7(5.4)$ & $15.5(3.3)$ \\
Years Driving & & $21.9(5.4)$ & $44.7(11.0)$ \\
Trail A (sec) & & $20.6(6.0)$ & $35.2(11.5)$ \\
$\bullet$ percentile & $87.4(13.0)$ & $81.7(16.1)$ \\
Trail B (sec) & & $46.0(13.6)$ & $84.0(11.6)$ \\
$\bullet$ percentile & & $84.1(16.4)$ & $83.7(11.6)$ \\
DSST & & $70.5(9.8)$ & $53.7(10.6)$ \\
UFOV & & & \\
$\bullet$ Processing speed & & $17.5(4.5)$ & $38.7(61.4)$ \\
$\bullet$ Divided attention & & $23.2(23.1)$ & $90.2(100.9)$ \\
$\bullet$ Selective attention & & $78.6(33.6)$ & $201.2(97.6)$ \\
\hline
\end{tabular}

of the cognitive/perceptual screening tests; however participants all fell within normal limits when compared to the age-related norms of each assessment.

Of the 54 participants, two senior women required an altered route when driving with hand-controls as it was considered too dangerous (by the drivers and the evaluators) to progress to highway driving and higher traffic densities. The 91-year-old gentleman attempted the hand-controls in the parking lot, but was not considered safe to enter traffic without pursuing supplementary training. His scores were therefore omitted from the data analysis.

The predicted driving risks, according to scores obtained on the UFOV, are presented for younger and older drivers (Figure 1). With the exception of five older drivers, all volunteers were categorized as very low or low risk for driving. Only two seniors were considered moderate-high risk for driving, and these were among the oldest volunteers in the study (79- and 91-years-old). 
FIGURE 1. Useful Field of View (UFOV) predicted driving risk.

\section{UFOV Risk}

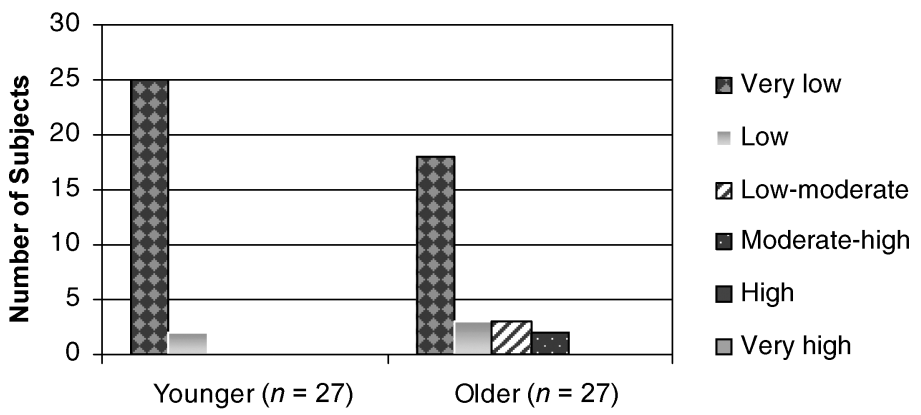

Age Category

\section{Impact of Introducing an Adaptive Device}

The mean scores in each of the six subscales on the NASA TLX for the entire route were significantly higher when driving with hand-controls compared to the normal driving condition (Table 2). Participants rated mental demand as the biggest contributing factor to their overall workload once the hand-controls were implemented.

The ranks of overall workload for each of the four driving segments differed significantly between the two driving conditions $(p<.0001$ at each route segment and over the entire route) (Figure 2). Despite the nonthreatening environment of the empty parking lot, it is evident that overall perceived workload was highest upon the initial introduction of the hand-controls in segment A and gradually decreased as drivers progressed through the driving route.

TABLE 2. Comparison of Workload Subscales:

Hand-Control Versus Normal Driving

\begin{tabular}{lccc}
\hline Workload $(N=53)$ & $\begin{array}{c}\text { Normal Driving } \\
\text { M (SD) }\end{array}$ & $\begin{array}{c}\text { Hand-control } \\
\text { M (SD) }\end{array}$ & $\mathrm{P}$ \\
\hline Mental & $15.3(11.3)$ & $48.2(22.9)$ & $<0.0001$ \\
Physical & $12.3(10.2)$ & $42.3(22.9)$ & $<0.0001$ \\
Temporal & $13.0(10.6)$ & $36.3(22.1)$ & $<0.0001$ \\
Perceived performance & $18.2(14.1)$ & $41.6(21.9)$ & $<0.0001$ \\
Frustration & $11.5(9.2)$ & $35.1(22.1)$ & $<0.0001$ \\
Perceived safety & $10.9(9.5)$ & $33.7(20.2)$ & $<0.0001$ \\
OVERALL WORKLOAD & $13.6(9.4)$ & $39.5(19.9)$ & $<0.0001$ \\
\hline
\end{tabular}


FIGURE 2. Calculated overall workload per driving segment. Workload was significantly greater $(p<0.0001)$ at all segments and over the whole route while using the hand-controls compared to normal driving without hand-controls.

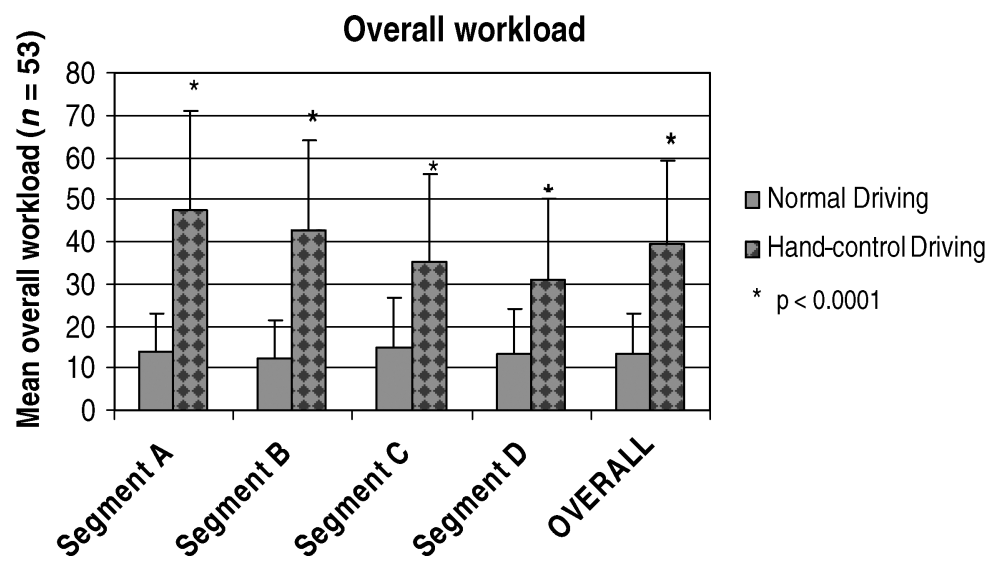

Comparison of the average speed indicated that although not dramatic, speed reduction was statistically significant for each segment when driving with adaptive equipment (Table 3 ).

\section{Age and the Introduction of an Adaptive Device}

Workload ratings on each subscale of the NASA TLX for the older and the younger drivers are presented in Figure 3.

Although task demand was perceived as slightly higher in the older group, for those who completed the protocol in its entirety, the change in workload (hand-control driving-normal driving) was not significantly

TABLE 3. Comparison of Speed in Normal Driving and Hand-Control Driving

\begin{tabular}{lccc}
\hline & $\begin{array}{c}\text { Change in speed (km/hr) } \\
\text { (normal - hand-control driving) } \\
\text { Mean (SD) }\end{array}$ & $\begin{array}{c}\text { Change in speed (km/hr) } \\
\text { Interquartile range }\end{array}$ & $p$ \\
\hline Segment A & $0.9(1.3)$ & $0-2.0$ & $<0.0001$ \\
Segment B & $2.5(3.1)$ & $0-4.0$ & $<0.0001$ \\
Segment C & $2.5(2.5)$ & $-0.5-5.5$ & $<0.0002$ \\
Segment D & $1.8(4.5)$ & $0.0-3.0$ & $<0.0003$ \\
\hline
\end{tabular}


FIGURE 3. Mean scores of workload subscales-younger vs. older drivers. Younger and older drivers did not have significantly different workload ratings during normal driving, nor during hand-control driving.

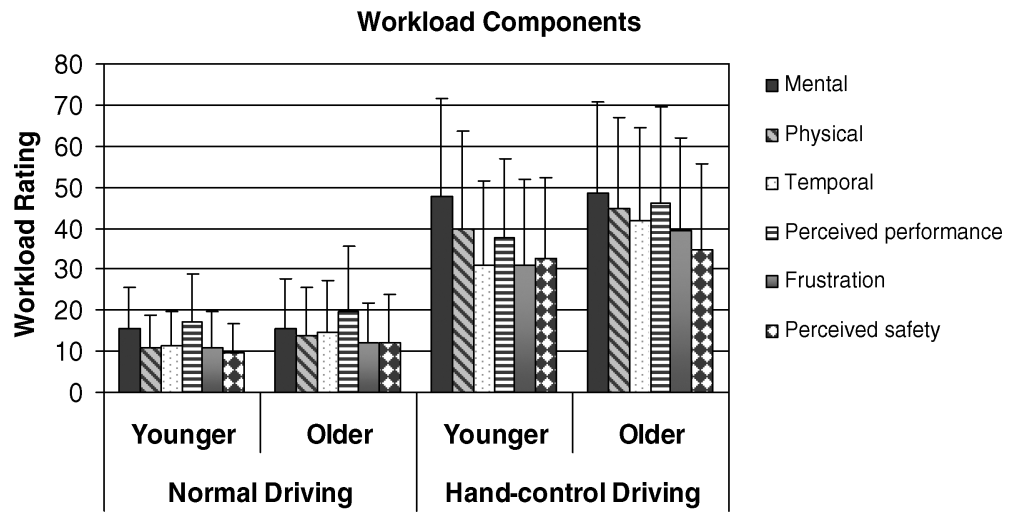

different between the older and younger cohorts for any of the workload subscales (Table 4).

\section{Association Between Sociodemographic Characteristics and Perceptual/Cognitive Status with Perceived Workload}

There were no significant associations between change in perceived workload and driving experience $(r=0.075)$, mileage $(r=0.07)$, or scores obtained on any of the three cognitive-perceptual screening evaluations (DSST: $r=-0.153$; UFOV processing speed: $r=0.131$, divided

TABLE 4. Older and Younger Drivers' Change in Perceived Workload

\begin{tabular}{lccc}
\hline & \multicolumn{3}{c}{ Mean change in workload } \\
\cline { 2 - 4 } Workload Subscales & Younger $(N=27)$ & Older $(N=26)$ & $p$ \\
\hline Mental & $26.6(56.2)$ & $27.4(56.2)$ & 0.8657 \\
Physical & $26.3(56.1)$ & $27.8(56.1)$ & 0.7351 \\
Temporal & $23.9(56.2)$ & $30.2(56.2)$ & 0.1395 \\
Perceived performance & $24.5(56.1)$ & $29.6(56.1)$ & 0.2397 \\
Frustration & $23.9(56.1)$ & $30.2(56.1)$ & 0.1443 \\
Perceived safety & $27.1(56.2)$ & $26.8(56.2)$ & 0.9503 \\
OVERALL WORKLOAD & $25.3(56.2)$ & $28.7(56.2)$ & 0.4283 \\
\hline
\end{tabular}


FIGURE 4. Gender comparison of perceived workload. In the normal driving condition there were no significant differences in perceived workload between the men and women, however, in the hand-control driving condition, the women had a significantly greater workload rating than the men.

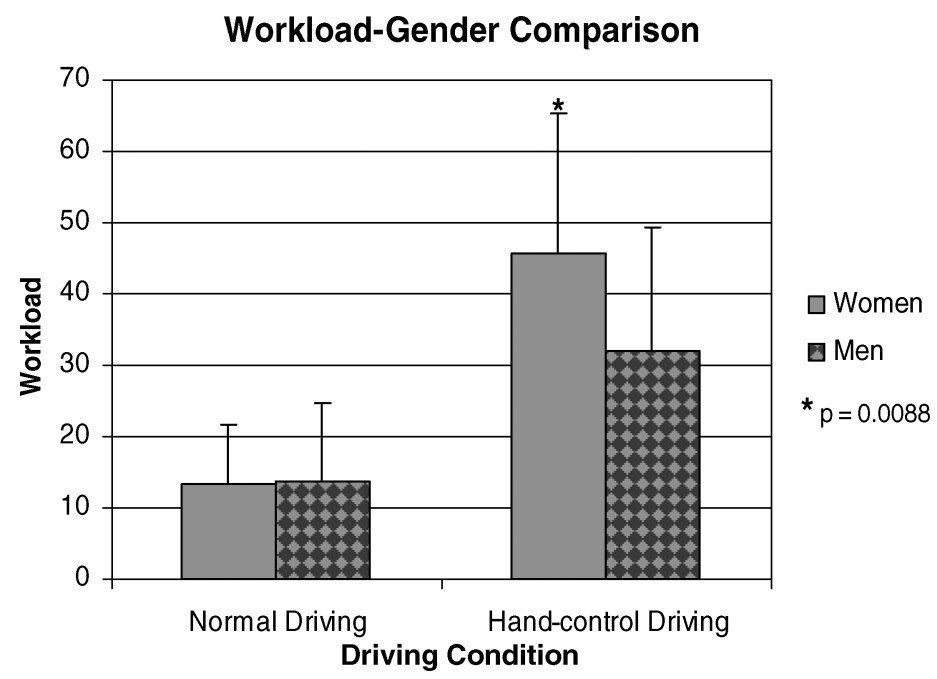

attention: $r=0.074$, selective attention: $r=0.0653$; Trail A: $r=0.0403$, Trail B: $r=0.0321$ ).

When comparing the results with respect to gender, ratings of task demand in the normal driving condition are almost identical for men and women (Figure $4 ; p>.6$ ). However, the introduction of hand-controls is perceived as significantly more demanding by women drivers $(p=.01)$, despite this group being younger than the men in both the older and younger groups (Table 1). Change in workload (hand-control-normal driving) was also significantly different between male and female participants ( $p=$ $.009)$.

\section{DISCUSSION}

The findings of the study clearly indicate that the introduction of handcontrols is an anxiety-provoking experience for drivers, greatly increasing the perceived workload of the driving task. This was consistent across both age cohorts. The implementation of an unfamiliar device to activate a vehicle's controls may therefore be considered as a cognitive 
distracter, imposed onto an already complex and demanding task. The significant increases in perceived workload reported by drivers when the adaptive equipment was introduced supports findings from other studies analyzing the impact of cognitive load on the driving task (Harbluk \& Noy, 2002; Lansdown et al., 2004; Recarte \& Nunes, 2003) and suggest that further training is indicated to return drivers to baseline workload levels.

Results did not indicate any association between sociodemographic factors and perceived workload, with the exception of gender. Although the women as a group were younger than the men, their change in workload increased significantly with the introduction of the adaptive equipment. Perceptual/cognitive status was not associated with change in perceived workload.

As recruitment was conducted through personal contacts and via wordof-mouth referrals, the volunteers in both age cohorts were very active, highly educated individuals. Participants tended to be confident and skilled drivers. During recruitment of the older drivers particularly, persons feeling less comfortable behind-the-wheel simply did not agree to participate in the study. The results obtained therefore reflect fairly ideal circumstances; drivers were healthy, active individuals with good driving habits and a high level of confidence prior to the introduction of adaptive equipment. This contrasts quite significantly to the clients typically evaluated in clinic presenting with functional deficits. These clients often experience increased stress in the testing environment due to potential loss of driving privileges following the assessment. It is therefore likely that the perceived workload for clients assessed in the context of rehabilitation would be magnified even further due to their contextual circumstances.

When analyzing the scores obtained on the NASA TLX, it is evident that the dimension of "mental demand" was considered to be the aspect of perceived workload most affected by the introduction of hand-controls. This likely reflects a decrease in the drivers' ability to rely on well-developed automatisms and motor patterns that have been integrated within their "normal" driving behaviors. When the task is modified at the operational level, a driver is forced to attend to the activation of the vehicle's controls, imposing greater demands on his attentional resources. The perceived physical demand while driving with hand-controls was also quite high. Surprisingly, this was largely due to the unilateral manipulation of the steering wheel. Although a spinner knob was provided to facilitate turning, one of the two vehicles used in the study appeared to have a much "stiffer" steering than the other. Clients using this vehicle commented on the physical demands of turning and scored this dimension on the rating scale as highly demanding. 
As it was not anticipated that the vehicles would differ to such a degree, this information was not systematically recorded in the data collection.

The driving route was planned to progress toward increasingly demanding traffic environments. Despite this, drivers reported less overall workload as they progressed to each segment of the route. While task demands remained quite high following the final segment of the driving course, this gradual decline indicates that the drivers started adapting to the new driving devices quite quickly.

Consistent with other findings (Harbluk \& Noy, 2002; Lansdown et al., 2004), the subjects decreased their speed when the hand-control was introduced into the driving task. This is likely a compensatory reaction, indicating a drivers' reluctance to drive while in a state of increased anxiety, when perceived workload is high. In an effort to reduce the task demands, drivers tend to slow down, thus increasing the time available to respond to the traffic environment. The Davis Carchip ${ }^{\mathrm{TM}}$ was used to measure speed variations, and to record the incidence of hard braking and accelerating. Analysis of the speed data compared specific segments in "normal" driving to the same segment in "hand-control" driving. Although incidences of hard accelerations and hard braking were recorded, the manner in which the hand-control was installed onto the vehicle's pedals influenced the sensitivity of the device to braking or accelerating. As the installation varied with each participant, the data obtained with respect to hard braking and hard accelerating was not considered reliable and was therefore not included in the analysis.

When comparing the older drivers to their younger counterparts, it was remarkable that on the six subscales of the NASA TLX, the profile of the senior drivers in the "normal" driving condition was almost identical to that of the younger group. Again, when the adaptive equipment was introduced, the younger and older participants responded very similarly. This outcome was surprising, as it was expected that slower information processing speeds and decreased attentional abilities would have a greater impact on the older group compared to their younger counterparts. A possible explanation may be the profile of the participating older drivers, who were relatively young (21/27 were in their sixties), healthy and very active. Results on the cognitive-perceptual screening tests ranked this group above the 80th percentile for their age in both the Trail A and Trail B tests, indicating that age-related decline in attentional abilities was not as evident in this sample compared to that found in the general population. Scores on the UFOV visual attention analyzer were also very good, with $22 / 27$ seniors falling in either the "very low" or the "low" driving risk categories. 
The analysis did not indicate any overall associations between change in perceived workload and perceptual/cognitive status. However, post hoc analysis by category on the UFOV visual attention analyzer suggest otherwise. Of the three seniors scoring in the low-moderate category on the UFOV, one completed the test without difficulty, one completed the test but reported extreme anxiousness, and the third participant was also anxious and was unable to complete the highway segment due to safety concerns. The UFOV categorized two of the older seniors as moderate-high risk behind the wheel. Neither of these participants were able to enter the highway although there is not enough data to infer conclusions to the general population, of five older drivers scoring more than a "low" risk on the UFOV, only one was able to complete the driving route without difficulty. These findings are not surprising as attention ability is a key factor in the capacity to learn a new task (Studer, 2004).

With respect to gender, the analysis revealed a greater change in perceived workload (hand-control driving-normal driving) for the women when compared to the men in the study. Although this was not an anticipated outcome, gender differences became evident during the data collection process. Drivers often verbalized their pre-occupations and anxieties when the hand-controls were introduced. The subjective impressions of the evaluators indicated that the female participants expressed more anxiety, especially with respect to the highway-driving component. In fact, many women expressed their concerns regarding the highway (segment $\mathrm{C}$ of the route) within the first $5 \mathrm{~min}$ of hand-control driving, while still in the empty parking lot (segment A). Interestingly, research on gender and communication reveals many differences between males and females, as language is reflective of social values and perceived roles (Mulvaney, 1994). It is possible that the reporting of anxiety and apprehension (both verbally and via the NASA TLX) was simply more readily communicated by the women in the study than the men.

\section{CONCLUSIONS}

The results of this study clearly indicate that modifying the controls of a vehicle greatly increases a driver's perceived workload of the driving task. Although drivers are able to start integrating adaptive equipment into their driving habits fairly quickly, task demands remain high even after completing a 30-kilometer route with a driving instructor. This validates the need for formal driver retraining when adaptive equipment is recommended for clients presenting with physical impairments. Healthy, active 
seniors responded similarly to younger drivers when hand-controls were introduced, but those with more clinically evident age-related attentional declines experienced additional difficulties. Although men and women perceived task demands of the normal driving condition similarly, gender differences were observed in ratings of perceived workload when unfamiliar devices were introduced. This may indicate that women drivers would benefit from a longer training period than men in order to fully integrate adaptive equipment into their driving habits and to reduce anxiety.

These findings suggest that the introduction of an adaptive device increases a driver's perceived workload, indicating that retraining is necessary for both younger and older drivers. Research is lacking with respect to its effect, and whether gender and age impact the amount of driver training required.

\section{ACKNOWLEDGMENTS}

The authors thank the participants of the study for their outstanding time sacrifices and commitment. Much gratitude to the driving instructors (Mario Champagne and Raymond Charette) and the occupational therapists (Carolle Leroux, Nathalie Maertens, and Ginette Lavigne) involved in the data-collection phase, and to Gevorg Chilinganyan whose guidance during the statistical analysis was pivotal.

This project was undertaken within the scope of a Master's Degree project and was funded by the Québec Rehabilitation Research Network (REPAR). A research fellowship was generously provided by the Canadian Driving Research Initiative for Vehicular Safety in the Elderly (CanDrive), funded by a New Emerging Team Grant from the Canadian Institutes of Health Research (CIHR). This paper was presented at the Canadian Association of Occupational Therapists Conference, Evidence and Occupation: Building the Future, June 2006, Montréal, Canada.

\section{REFERENCES}

Anderson, N.D. (1999). The attentional demands of encoding and retrieval in younger and older adults: Evidence from secondary task reaction time distributions. Psychological aging, 14(4), 645-655.

Ball, K., Owsley, C., Sloane, M.E., Roenker, D.L., \& Bruni, J.R. (1993). Visual attention problems as a predictor of vehicle crashes in older drivers. Investigative Ophthalmology and Visual Science, 34(11), 3110-3123. 
DeWaard, D. (1996). The measurement of drivers' mental workload. PhD thesis, University of Groningen. Haren, The Netherlands: University of Groningen, Traffic Research Centre. 27.

Dickman, S.S., Heaton, R.K., Grant, I., \& Temkin, N.R. (1999). Test-retest reliability and practice effects of expanded halstead-reitan neuropsychological test battery. Journal of the International Neuropsychological Society, 5, 346356.

Drane, D.L.P.D., Yuspeh, R.L.P.D., Huthwaite, J.S.M.A., \& Klingler, L.K.M.A. (2002). Demographic characteristics and normative observations for derived-trail making test indices. Neuropsychiatry, Neuropsychology, and Behavioral Neurology, 15(1), 39-43.

Dulisse, B. (1997). Older drivers and risk to other road users. Accident Analysis and Prevention, 29, 573-582.

Golde, T. (2001). What cognitive changes take place with age? Biology of Aging. Retrieved August 15, 2006, from http://www.healthandage.com/html $/ \mathrm{min} /$ afar/content/other6_1.htm.

Harbluk, J., \& Noy, I. (2002). The impact of cognitive distraction on driver visual behaviour and vehicle control. (No. TP\# 13889E): Road Safety and Motor Vehicle Regulation Directorate, Transport Canada, Moshe Eizenman, EL-MAR Inc.

Hart, S.G., \& Staveland, L.E. (Eds.). (1988). Development of NASA-TLX (Task Load Index): Results of empirical and theoretical research. Amsterdam: North-Holland.

Henriksson, P., \& Peters, B. (2004). Safety and mobility of people with disabilities driving adapted cars. Scandinavian Journal of Occupational Therapy, 11, 54-61.

Hill, S., Iavecchia, H., Byers, J., \& Bittner, A. (1992). Comparison of four subjective workload rating scales. Human factors, 34(4), 429-439.

Hopewell, C.A. (2002). Driving assessment issues for practicing clinicians. Journal of Head Trauma Rehabilitation, 17, 48-61.

Hoyer, W., Stawski, R., Wasylyshyn, C., \& Verhaeghen, P. (2004). Adult age and digit symbol substitution performance: A meta-analysis. Pyschology and Aging, 19(1), 211-214.

Huebner, K., Porter, M.M., \& Marshall, S.C. (2006). Validation of an electronic device for measuring driving exposure. Traffic Injury Prevention, 7, 76-80.

Lansdown, T.C., Brook-Carter, N., \& Kersloot, T. (2004). Distraction from multiple invehicle secondary tasks: Vehicle performance and mental workload implications. Ergonomics, 47(1), 91-104.

Legree, P., Heffner, T., Psotka, J., Martin, D., \& Medsker, G. (2003). Traffic crash involvement: Experimental driving knowledge and stressful contextual antecedents. Journal of Applied Psychology, 8(1), 15-26.

Lyman, S., Ferguson, S., Braver, E., \& Williams, A. (2002). Older driver involvements in police reported crashes and fatal crashes: Trends and projections. Injury Prevention, 8, 116-120.

Mazer, B., Gélinas, I., \& Benoit, D. (2004). Evaluating and retraining driving performance in clients with disabilities. Critical Reviews in Physical and Rehabilitation Medicine, 16(4), 293-328. 
Monga, T.N. (1997). Driving: A clinical perspective on rehabilitation technology. Physical Medicine and Rehabilitation, 11(1), 69-92.

Mulvaney, B.M. (1994). Gender differences in communication: An intercultural experience. Retrieved August 24, 2006, from http://ksuweb.kennesaw.edu/ $\sim$ mwalter1/Gender\%20Differences.htm.

National Highway Traffic Safety Administrations (NHTSA). (2003). Model driver screening and evaluation program: Final technical report, volume I: Project summary and model program recommendations. Retrieved October 14, 2008, from http://www.nhtsa.dot.gov/people/injury/olddrive/modeldriver/1_chap_1.htm.

Nygren, T. (1991). Psychometric properties of subjective workload measurement techniques: Implications for their use in the assessment of perceived mental workload. Human Factors, 33(1), 17-33.

O’Neill, D. (2002). Safe mobility for older people. Department for Transport, London, U.K. (Unpublished manuscript)

O’Neill, D., Bruce, I., Kirby, M., \& Lawlor, B. (2000). Older drivers, driving practices and health issues. Clinical Gerontologist, 22, 47-54.

Owsley, C., Ball, K., McGwin, G., Sloane, M.E., Roenker, D.L., White, M.F., et al. (1998). Visual processing impairment and risk of motor vehicle crash among older adults. Journal of the American Medical Association, 279(14), 1083-1088.

Parkin, A., \& Java, R. (1999). Deterioration of frontal lobe function in normal aging: Influences of fluid intelligence versus perceptual speed. Neuropsychology, 13(4), 539-545.

Pavlik, V., Alves de Moraes, S., Szklo, M., Knopman, D., Mosley, T., \& Hyman, D. (2003). Relation between cognitive function and mortality in middle-aged adults. American Journal of Epidemiology, 157, 327-334.

Perez Riley, K. (Ed.). (1994). Cognitive development. Philadelphia: F.A. Davis.

Persson, D. (1993). The elderly driver: Deciding when to stop. Gerontologist, 33(1), 88-91.

Peters, B. (2001). A framework for evaluating adapted passenger cars for drivers with physical disabilities. Linköping University, Sweden, Linköping.

Recarte, M., \& Nunes, L. (2003). Mental workload while driving: Effects on visual search, discrimination, and decision making. Journal of Experimental Psychology: Applied, 9(2), 119-137.

Rubio, S., Diaz, E., Martin, J., \& Puente, J. (2004). Evaluation of subjective mental workload: A comparison of SWAT, NASA TLX, and workload profile methods. Applied Psychology: An International Review, 53(1), 61-86.

Sekuler, A., Bennett, P., \& Mamelak, M. (2000). Effects of aging on the useful field of view. Experimental Aging Research, 26, 103-120.

Spreen, O., \& Strauss, E. (1991). A compendium of neuropsychological tests. New York: Oxford University Press.

Studer, M. (2004). Cognitive rehabilitation in the frail elderly patient: Never too old to learn? Topics in Geriatric Rehabilitation, 20(1), 21-33.

Summala, H. (1988). Risk control is not risk adjustment: The zero-risk theory of driver behavior and its implications. Ergonomics, 31, 491-506.

Tomporowski, P. (2003). Performance and perceptions of workload among young and older adults: Effects of practice during cognitively demanding tasks. Educational Gerontology, 29, 447-466. 
Tuokko, H., \& Hunter, F. (2002). Using "age" as a fitness-to-drive criterion for older adults. Retrieved October 13, 2003 from http://www.lcc.gc.ca/ en/themes/pr/oa/tuokko/toc.asp.

Turnbull, A., \& McKenzie, J. (1997). Persons with disabilities and converted vehicles. Phase I: Preliminary investigation. Phase II: Detailed study design. Retrieved October 20, 2008, from http://www.tc.gc.ca/innovation/tdc/summary/ 13000/130193.htm.

Verway, W.B. (1992). On evaluating vehicle adaptations for disabled drivers (No. IZF 1992 C-36). Soesterberg, The Netherlands: TNO Road Vehicles Research Institute.

Wechsler, D. (1981). Manual for the Wechsler adult intelligence scale-revised. New York: The Psychological Corporation.

Received: 06/16/08

Revised: 01/19/09

Accepted: 02/02/09 